\title{
A new Wolf-Rayet star in Cygnus ${ }^{\star}$
}

\author{
A. Pasquali ${ }^{1}$, F. Comerón ${ }^{2}$, R. Gredel ${ }^{3}$, J. Torra ${ }^{4}$, and F. Figueras ${ }^{4}$ \\ 1 ESO/ST-ECF, Karl-Schwarzschild-Strasse 2, 85748 Garching bei München, Germany \\ 2 ESO, Karl-Schwarzschild-Strasse 2, 85748 Garching bei München, Germany \\ e-mail: fcomeron@eso.org \\ 3 Max-Planck Institut für Astronomie, Königstuhl 17, 69117 Heidelberg, Germany \\ e-mail: gredel@caha.es \\ 4 Departament d'Astronomia i Meteorologia, Universitat de Barcelona, Av. Diagonal 647, 08028 Barcelona, Spain \\ e-mail: jordi@am.ub.es; cesca@am.ub.es
}

Received 22 August 2002 / Accepted 19 September 2002

\begin{abstract}
We report the discovery of a new Wolf-Rayet star in the direction of Cygnus. The star is strongly reddened but quite bright in the infrared, with $J=9.22, H=8.08$ and $K_{S}=7.09$ (2MASS). On the basis of its $H+K$ spectrum, we have classified WR 142a a WC8 star. We have estimated its properties using as a reference those of other WC8 stars in the solar neighbourhood as well as those of WR 135, whose near-infrared spectrum is remarkably similar. We thus obtain a foreground reddening of $A_{V} \simeq 8.1 \mathrm{mag}, M_{J} \simeq-4.3, \log \left(L / L_{\odot}\right) \sim 5.0-5.2, R=0.8 R_{\odot}, T \simeq 125000 \mathrm{~K}, M=7.9-9.7 M_{\odot}, \dot{M}=(1.4-2.3) \times 10^{-5} M_{\odot} \mathrm{yr}^{-1}$. The derived distance modulus, $D M=11.2 \pm 0.7 \mathrm{mag}$, places it in a region occupied by several $\mathrm{OB}$ associations in the Cygnus arm, and particularly in the outskirts of both Cygnus OB2 and Cygnus OB9. The position in the sky alone does not allow us to unambiguously assign the star to either association, but based on the much richer massive star content of Cygnus OB2 membership in this latter association appears to be more likely.
\end{abstract}

Key words. stars: emission line, Be - stars: fundamental parameters - stars: Wolf-Rayet Galaxy: open clusters and associations: general

\section{Introduction}

Wolf-Rayet (WR) stars, first discovered by Wolf and Rayet in 1867 , are characterised by an emission-line spectrum dominated by strong $\mathrm{He}, \mathrm{N}$ and $\mathrm{C}$ features. Their observed properties are believed to be the result of stellar evolution at high initial masses, during which strong mass loss in winds of a few $10^{-5} M_{\odot} \mathrm{yr}^{-1}$ at terminal velocities between 400 and $5000 \mathrm{~km} \mathrm{~s}^{-1}$ (cf. van der Hucht 2001) "peels" the star until it comes to expose at its surface the heavy elements synthesized in its core. WR stars represent the last evolutionary stage of massive stars before the final supernova explosion (Maeder \& Conti 1994; Langer \& Woosley 1996; Langer \& Hegel 1999). The flux ratios among the $\mathrm{He}, \mathrm{N}$, and $\mathrm{C}$ lines allows the classification of WR stars into subclasses, WN (where He and N lines dominate), $\mathrm{WC}$ and $\mathrm{WO}$ (when $\mathrm{He}, \mathrm{C}$ and $\mathrm{O}$ features become stronger), and subtypes (WN3-9 and WC4-9 as the ionization degree increases).

Send offprint requests to: A. Pasquali, e-mail: apasqual@eso.org

* Based on observations collected at the German-Spanish Astronomical Centre, Calar Alto, operated by the Max-PlanckInstitut für Astronomie, Heidelberg, jointly with the Spanish National Commission for Astronomy.
WR stars significantly contribute to the energy budget and chemical enrichment of their parent galaxy. Through their stellar winds, they enrich the hosting environment with heavy elements and release over time a kinetic energy comparable to that from supernovae (Leitherer 1998). Given their high luminosities (few $10^{5} L_{\odot}$ ) individual WR stars are easily identified in all the galaxies of the Local Group (Massey \& Johnson 1998), while the short lifetimes of such massive stars (several Myr) make them appear generally near their birthplaces, tracing star formation in spiral and starburst galaxies. Indeed, their unmistakable features, detected in the integrated spectra of galaxies undergoing intense bursts of star formation (cf. Conti 2000), make them useful to study starburst phenomena and star formation processes on cosmological scales.

The latest census of WR stars detected in the Milky Way lists 227 such objects (van der Hucht 2001), or about 10\% of the estimated Galactic WR population $(\simeq 1500-2500$; Shara et al. 1999). The majority of Galactic WR stars has escaped detection using traditional techniques (such as visible spectroscopy or narrow-band photometry with filters centered on the characteristic WR signature at $\lambda \simeq 4670 \AA$ and its adjacent continuum, cf. Massey \& Conti 1983) which are naturally limited by their unability to penetrate the large column densities 
of absorbing dust along the lines of sight towards distant regions of the galactic disk. Such limitation can be overcome by observing at near-infrared wavelengths, where WR stars also display easily identifiable spectral signatures and the extinction by dust is only a fraction of that at shorter wavelengths.

In this paper we report the discovery of a new nearby WR star, supporting the prediction by van der Hucht (2001) that many such stars in the solar neighbourhood still await discovery at near-infrared wavelengths. Indeed, the object reported here is bright at near-infrared wavelengths but has escaped recognition so far in the visible despite its proximity, due to the high extinction in its direction. The discovery of yet another nearby WR star deserves attention in several respects, as each WR star has its own special features and represents a unique contribution to our understanding of the late stages of massive stellar evolution. Moreover, its location and relation to other young stars in the region yields information on the star formation history in the complex where it formed. The near-infrared observations of the star, to which we will refer as WR 142a following the nomenclature in van der Hucht's (2001) catalogue, are described in Sect. 2. The results concerning its spectral classification and intrinsic properties can be found in Sect. 3 . Finally, Sect. 4 discusses some implications of the WR population in Cygnus.

\section{Observations}

The selection of WR 142a as a target for spectroscopic observations was based on its 2MASS photometry, from which it was apparent that this star is a bright, red object with colours significantly deviating from those of normal stars reddened by foreground dust. Infrared magnitudes listed by 2MASS are $J=9.22, H=8.08, K_{S}=7.09$. Its location at $\alpha(2000)=$ $20^{\mathrm{h}} 24^{\mathrm{m}} 06.2^{\mathrm{s}}, \delta(2000)=+41^{\circ} 25^{\prime} 33^{\prime \prime}$ places it in the general direction of Cygnus, in a region where several OB associations overlap (see Sect. 3.4). The USNO catalog lists a star of $B=18.1, R=14.7$ at the position of this object. The only previous reference to it in the literature seems to be Melikian \& Shevchenko (1990), who recognized it as a strong emissionline star in their search for such objects in the proximities of the cluster NGC 6910. However, its actual WR nature has not been recognized until now.

We observed WR142a on June 24 and 25 2002, using the near-infrared imager and spectrograph MAGIC mounted on the $1.23 \mathrm{~m}$ telescope of the German-Spanish Astronomical Center in Calar Alto (Spain). This instrument uses a NICMOS3 $256 \times 256$ pixel $^{2}$ detector yielding a scale of 1 .' 15 per pixel in imaging mode. $J H K_{M}$ imaging and $H$ and $K$ band spectroscopy were obtained and reduced using the same instrumental setups and techniques described in Comerón et al. (2002); the reader is thus referred to that paper for details. Two spectra obtained on the night of 24 June 2002 at airmasses of 2.05 and 1.67 were individually reduced to confirm the reality of individual features, especially in regions of rapidly varying telluric absorption. The exposure time of the combined spectrum is $12 \mathrm{~min}$. As for the imaging, the exposure times are $7.5 \mathrm{~min}$ in each of the $J, H$ and $K_{M}$ filters. The images were obtained under conditions suspected to be non-photometric, and flux

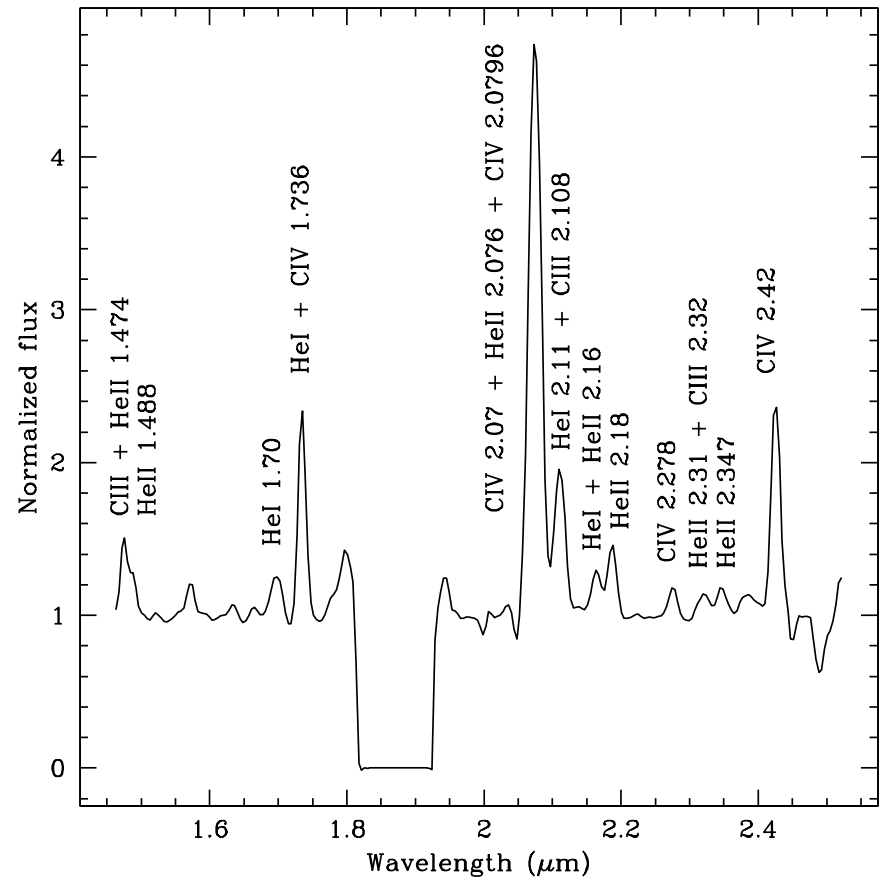

Fig. 1. The observed $H$-to- $K$ spectrum of WR 142a. The spectral resolution is $R \simeq 240$. The drop between 1.8 and $1.9 \mu \mathrm{m}$ is due to the Earth atmosphere transmission.

calibration using a separate image of a standard star was thus not attempted.

\section{Results}

\subsection{IR spectroscopy and classification}

The low-resolution infrared spectrum of WR 142a $(R \simeq 240)$ is plotted in Fig. 1. Emission lines have been identified by cross-correlation with The Atomic Line List v2.04 (http://www.pa.uky.edu/ peter/atomic) and the $K$ band spectral atlas of Figer et al. (1997). The most prominent features are due to HeI and HeII, CIII and CIV. Their equivalent widths are listed in Table 1.

We have cross-checked the spectrum of WR 142a with the $K$-band spectral atlas of Galactic WR stars by Figer et al. (1997) in order to assign WR 142a a WR subtype. The line complex between 2 and $2.2 \mu \mathrm{m}$ has been particularly useful in the spectral classification, since it is a specific signature (in line wavelength and flux ratio) of WC8 stars (cf. Fig. 10 in Figer et al. 1997). Although the dispersion of Figer et al.'s (1997) data is a factor of two higher $(R \simeq 525$ against 240 of this work), the comparison indicates that the spectrum of WR 142a is almost identical to that of WR 135, a WC8 star in the Galactic OB association Cygnus OB3. And similarly to WR 135, a weak absorption can be detected blueward of the $\mathrm{CIV}+\mathrm{HeII}$ emission line at $\lambda=2.075 \mu \mathrm{m}$ suggesting a $\mathrm{P}$ Cygni profile for this line blend (or for the HeII $2.06 \mu \mathrm{m}$ feature buried in this line blend). Therefore, we classify WR 142a as a WC8 star. 
Table 1. Line equivalent widths in the IR spectrum of WR 142a.

\begin{tabular}{lc}
\hline \hline Line $(\mu \mathrm{m})$ & EqW $(\AA)$ \\
\hline CIII + HeII 1.474 & 50 \\
HeII 1.488 & 30 \\
HeI 1.70 & 60 \\
HeI + CIV 1.736 & 200 \\
CIV 2.07 + HeII 2.076 + CIV 2.08 & 780 \\
HeI 2.11 + CIII 2.108 & 160 \\
HeI + HeII 2.16 & 40 \\
HeII 2.18 & 70 \\
CIV 2.278 & 30 \\
HeII 2.31 + CIII 2.32 & 30 \\
HeII 2.347 & 30 \\
CIV 2.42 & 200 \\
\hline
\end{tabular}

\subsection{Colors, distance, reddening and variability}

As a first step towards the estimate of the physical properties of WR 142a, we have compared its near-infrared photometry to that of six other nearby WC8 stars (WR 53, WR 60, WR 77, WR 101, WR 117, and WR 135) selected from Williams et al. (1987). Stars with detected companions were excluded. WR 101 and WR 135 have JHK photometry also from 2MASS, and WR 113 from Pitault et al. (1983); in all these cases, all published magnitudes agree to better than $0.1 \mathrm{mag}$. Extinctions in the visible, $A_{V}$, are given for the six reference objects by van der Hucht (2001) based on their visible photometry. A seventh WC8 star, WR 102e, also has available 2MASS photometry. However, the fact that this star is located in the galactic center raises some concerns about its validity as a reference for comparison to the WR population in the solar neighbourhood. Besides the published JHK photometry we have also used the extinction given in van der Hucht catalogue, from which we derive intrinsic colours $(J-H)_{0}$, $(H-K)_{0}$ using the extinction law of Rieke \& Lebofsky (1985).

Assuming that the intrinsic colours of WR 142a are the same as the average of those of the reference stars, we obtain $(J-H)_{0}=0.28 \pm 0.25$. The uncertainty includes both the intrinsic scatter in colours among WC8 stars and possible errors in determining the extinction, which is generally done using $B V$ colours. The derived extinction for WR 142a is thus $A_{V}=8.1 \pm 2.3$, or $A_{J}=2.3 \pm 0.6$. Using the $K$ band to estimate the extinction introduces larger uncertainties due to the larger scatter in derived intrinsic $(H-K)_{0}$ colours among the reference objects, and we have preferred to exclude it for this purpose. A more accurate estimate is possible by using the measured $B=18.1$ in the USNO catalogue, keeping in mind that a possible error may be introduced by variability in the nonsimultaneous $B$ and $J H K$ observations. In that case, we have calculated $(B-V)_{0}=-0.14$ using the observed $(B-V)$ of the reference stars and dereddening them with the $A_{V}$ listed by van der Hucht (2001), using $A_{V} /\left(A_{B}-A_{V}\right)=3.1$, thus obtaining $(B-J)_{0}=0.42$. The observed $(B-J)=8.9$ thus implies $A_{V}=8.1$, practically identical to the value derived from $J-H$ but based on a much longer wavelength baseline. The uncertainty, mostly due to the scatter in $(B-J)_{0}$, is accordingly smaller and estimated to be $0.4 \mathrm{mag}$. We thus adopt $A_{V}=8.1 \pm 0.4$, or $A_{J}=2.3 \pm 0.1$, as the extinction towards WR 142a.

A similar procedure applied to the $(V-J)$ photometry of the reference stars yields an estimate of $(V-J)_{0} \simeq 0.56 \pm 0.24$ for WR 142a, again with the caveat of the non-simultaneity of the visual and infrared observations although the relatively small scatter that we find in $(V-J)_{0}$ suggests that this may not be an important factor. Proceeding with the $(V-J)_{0}$ given above and assuming for the visual absolute magnitude of WR 142a the mean value derived by van der Hucht (2001) for WC 8 stars, $M_{V}=-3.74$, we obtain $M_{J}=-4.30$. In this case, to the already noted uncertainty in $(V-J)_{0}$ we must add the scatter in $M_{V}$ found for these stars, which van der Hucht evaluates at $0.5 \mathrm{mag}$. Using the estimates of the extinction and the absolute magnitude we obtain a distance modulus of $D M=11.2 \pm 0.7$, corresponding to a distance of $1.8_{-0.5}^{+0.6} \mathrm{kpc}$.

An alternative possibility consists of using the close similarity between the spectra of WR 142a and WR 135 to assume that the intrinsic properties of both are identical, including intrinsic colours and absolute magnitudes. WR 135 is the bluest star in the reference sample both in $(J-H)_{0}$ and $(V-J)_{0}$. The brighter $M_{V}$ listed by van der Hucht (2001) is balanced by the bluer colours to give $M_{J}=-4.33$, practically identical to the value used in the above estimate. However, fitting the colours requires a higher extinction $\left(A_{V}=11.0\right.$ or $\left.A_{J}=3.1\right)$ and the distance modulus thus reduces to $10.2 \mathrm{mag}$, corresponding to a distance of $1.2 \mathrm{kpc}$, in the lower end of the range given above.

Despite of the fact that our observations were probably performed under non-photometric conditions as noted in Sect. 2, it is still possible to check for possible variability by comparing WR 142a to other 2MASS stars in the field. Such comparison suggests that WR 142a was slightly fainter when we observed it than when it was observed by 2MASS, with $\Delta J=0.08$, $\Delta H=0.07, \Delta K=0.25$. Only the significance of the $K$ variability is more than marginal, lying within the amplitude range of the long-term variability of Wolf-Rayet stars reported by Hackwell et al. (1979) and well below the rather extreme case described by Danks et al. (1983).

\subsection{Properties and environment}

The physical properties of WR 142a (mass $M$, radius $R$, temperature $T$ ) may be estimated with simple relations derived by Schaerer \& Maeder (1992) from their models of WNE/WC stars, with the caveat that these models are quite sensitive to the assumed morphology of the stellar wind, i.e. clumpiness, which may change the bolometric luminosity of WRs. The luminosity can be estimated by adopting for WR $142 \mathrm{a} M_{V}=-3.74$ as determined by van der Hucht (2001) for WC8 stars, and the average bolometric correction $B C_{V}=-4.12$ found by Nugis \& Lamers (2000) for the WC8 subgroup (taking into account their Eq. (6) to transform from narrow- to broad-band $V$ magnitude). Since $\log \left(L / L_{\odot}\right)=-0.4 \times\left(M_{V}+B C_{V}-M_{\odot}^{\mathrm{BOL}}\right)$, the total 
luminosity of WC8 stars turns out to be $\log \left(L / L_{\odot}\right)=5.04$. If the individual luminosity of WR 135 is adopted instead as more appropriate for WR $142 \mathrm{a}$, we obtain $\log \left(L / L_{\odot}\right)=5.24$.

Using Schaerer and Maeder's mass-luminosity relation,

$\log \left(L / L_{\odot}\right)=3.032+2.695 \log \left(M / M_{\odot}\right)-0.461\left(\log \left(M / M_{\odot}\right)\right)^{2}$

we obtain a mass between 7.9 and $9.7 M_{\odot}$ (assuming respectively $\log L / L_{\odot}=5.04$ and 5.24 as described above). Likewise, the stellar radius $R$ and temperature $T$ are given approximated by

$\log \left(R / R_{\odot}\right)=-1.845+0.338 \times \log \left(L / L_{\odot}\right)$

and

$\log (T)=4.684+0.0809 \times \log \left(L / L_{\odot}\right)$

from where we obtain $R \simeq 0.8 R_{\odot}, T \simeq 125000 \mathrm{~K}$, with little dependence on the choice between 5.04 or 5.24 for $\log L / L_{\odot}$. Finally, adopting Nugis \& Lamers' equation (24), which gives the stellar mass loss as a function of the stellar present mass, we derive for WR142a a mass-loss rate of $1.4 \times 10^{-5} M_{\odot} \mathrm{yr}^{-1}$ or $2.3 \times 10^{-5} M_{\odot} \mathrm{yr}^{-1}$, depending on which one of the masses given above is adopted. It must be stressed that the values above are a simple estimate of the stellar properties of WR142a based on average properties of WC8 stars, and an accurate model atmosphere fitting of its IR spectrum is indeed needed.

Although the winds of late-type WC stars are generally a source of warm dust (Smith \& Houck 2001; Chiar et al. 2001), no IRAS mid-infrared point source is detected near the position of WR 142a. This is somewhat unexpected, as the sensitivity of IRAS should enable detection of a typical late WC star within $7 \mathrm{kpc}$ from the Sun (Cohen 1995). Mid-infrared observations provide no evidence either for any extended structure at larger scales around WR 142a, as is sometimes found the neighbourhood of other WR stars (e.g. Miller \& Chu 1993; Saken et al. 1995; Pineault \& Terebey 1997; Gervais \& St-Louis 1999, and references therein). This does not completely rule out the possible existence of an extended nebula, as the wide range of ring nebula sizes around WR stars (Miller \& Chu 1993) and the intricate structure of thermal infrared emission in the general direction of Cygnus would naturally make the detection of such a nebula elusive. Near-infrared images of the immediate surroundings of WR 142a (Fig. 2) also show it to be rather inconspicuous, with no obvious nebulosity associated to it within the $5^{\prime}$ field of view of our images.

\subsection{Membership}

At the estimated distance of $\simeq 1.8 \mathrm{kpc}$, WR $142 \mathrm{a}$ lies in the extended complex of massive star forming regions composed by several OB associations and part of the molecular complex Cygnus X (e.g. Odenwald \& Schwartz 1989). Its location with respect to the three most nearby associations and the other WR stars identified within $5^{\circ}$ of it are shown in Fig. 3 .

The overlap among the associations and the difficulty in precisely establishing their boundaries (or even in establishing them as different entities) makes it difficult to unambiguously assign WR 142a to one of them. Adopting the distribution of

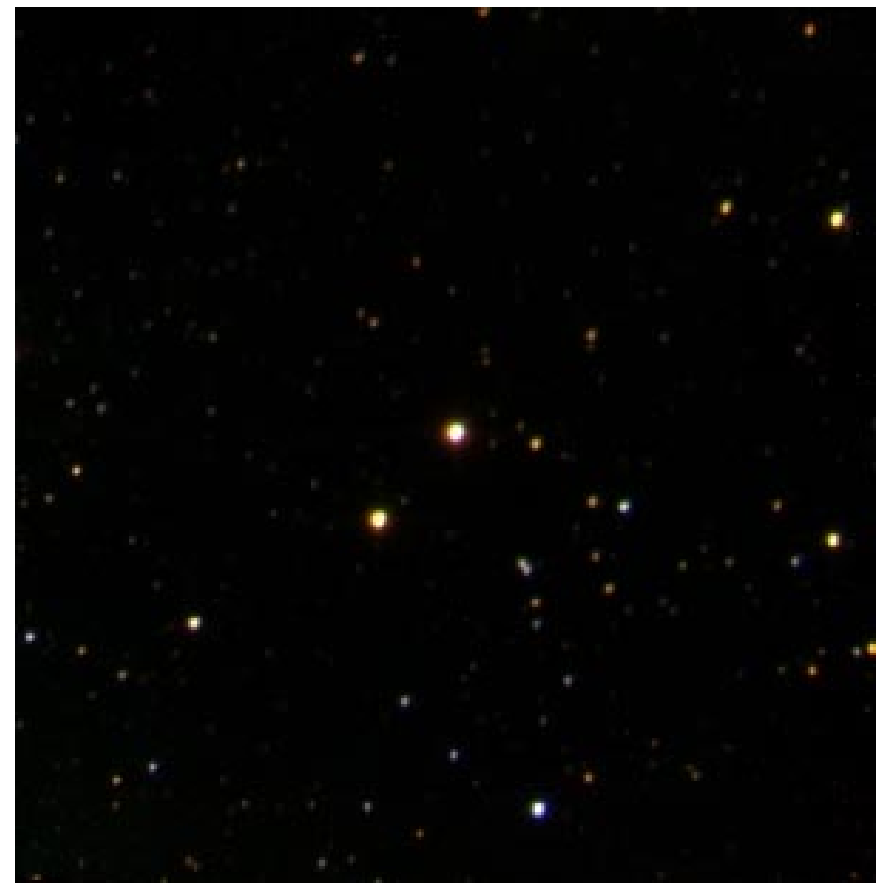

Fig. 2. A view of the field around WR 142a (the bright star at the center of the image) showing no evidence for extended emission. The printed version of this paper shows the $K$-band image, while a $J H K$ colour composite is presented in the electronic version.

members given by Garmany \& Stencel (1992), WR 142a lies near the boundaries of both Cygnus OB2 and Cygnus OB9. Association to the latter may be suggested in view of its proximity (less than $1^{\circ}$ ) to the open cluster NGC 6910. The average extinction towards Cygnus OB9, where members obscured by $A_{V}>6$ are listed by Garmany \& Stencel (1992), does not conflict with this assignment. It may be interesting to note that WR 142a appears in the sky about $10^{\prime}$ West of the compact HII region DWB87 (Dickel et al. 1969) and may be obscured by the molecular cloud surrounding it. No detailed studies of this nebula seem to exist in the literature, preventing us from ascertaining whether a physical connection between WR 142a and DWB87 may exist. On the other hand, it is also possible that WR 142a is actually a member of Cygnus OB2. Cygnus OB2 is a compact OB association (see Knödlseder 2000; Comerón et al. 2002, and references therein) rich in very massive members which are on the average more reddened than those of Cygnus OB9. Cygnus OB2 has four other WR stars, two of which also belong to the WC class, indicating progenitors with initial masses above $60 M_{\odot}$ (Crowther et al. 1995a), and in this respect the higher content of very massive stars of Cygnus OB2 favors the hypothesis that WR 142a is a member of this association lying in its outskirts. Given the considerable uncertainties affecting the definition of the membership, the boundaries, and the distances of each of these associations (see Comerón et al. 1998 for an additional discussion), the assignment of WR 142a to either Cygnus OB2 or Cygnus OB9 must remain as only tentative for the time being. 


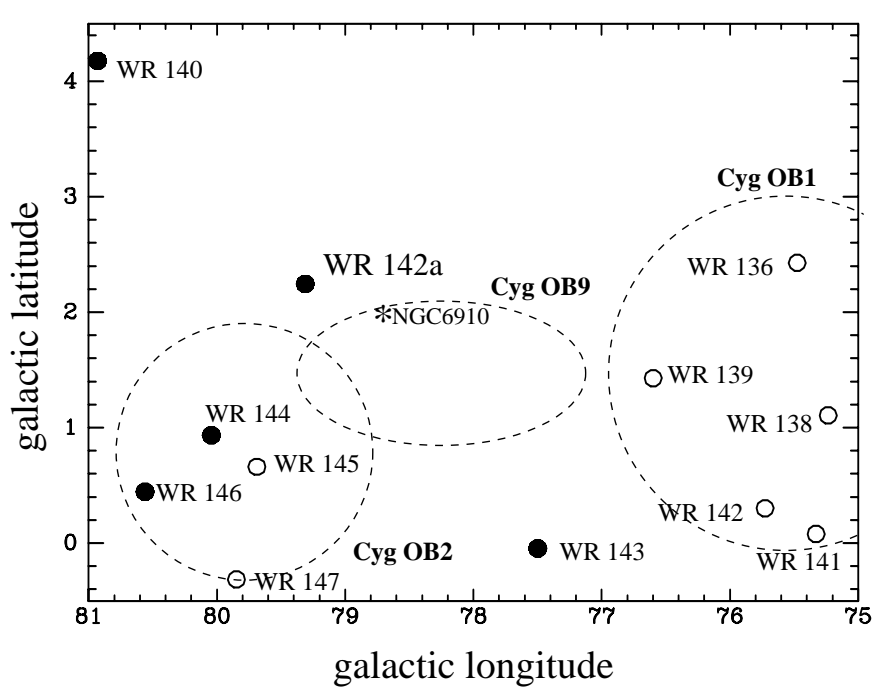

Fig. 3. Location of WR 142a with respect to the three OB associations in its neighbourhood. Also shown are the positions of other WR stars, with full circles representing WC stars and empty circles WN stars. The boundaries of the associations are a rough approximation to the distribution of their members as plotted in Garmany \& Stencel (1992).

\section{Discussion and conclusions}

Our near-infrared observations of a source in Cygnus with infrared colours deviating from those of normal, reddened stars has brought up the serendipitous discovery of another WR star in this complex of rich OB associations. Table 2 summarizes the parameters of this star, WR 142a.

The detection of WR 142a adds one more example of a late evolved star to the massive star content of this region. It is a remarkable fact that Cygnus OB2 contains examples of all the stages in the evolutionary path of the most massive stars, $\mathrm{O}$ main sequence $\rightarrow \mathrm{Of} / \mathrm{WN} \rightarrow \mathrm{LBV} \rightarrow \mathrm{WN} \rightarrow \mathrm{WC}$ for initial masses about $60 M_{\odot}$ (Langer et al. 1994). The addition of one more member of the rare class of stars with such high initial masses is thus a valuable one to a region that can be considered as the best avaliable laboratory for the observational study of stellar evolution at the upper end of the main sequence.

The abundant massive stars content of the complex Cygnus OB2/OB9, where over 100 O-type stars and descendants have been identified (Garmany \& Stencel 1992; Massey et al. 1995; Knödlseder 2000; Comerón et al. 2002), leads to the expectation that this complex may be at present or in the near future the scenario of intense supernova activity. Taking 7.1 Myr as the expected lifetime of a late O-type star (Meynet et al. 1994; Schaerer \& de Koter 1997), the content of Cygnus OB2/OB9 would lead to the expectation of a rate of about $1 \mathrm{SN}$ every less than $70000 \mathrm{yr}$. The observability of the radio-continuum signature of individual supernova remnants is limited in time and highly dependent on selection effects that are difficult to model (Green 1991), but the inferred interval between supernovae in Cygnus is rather short as compared to the estimated age of many supernova remnants (Matthews et al. 1998). Thus, the fact that none has been observed within 2 degrees from the centre of Cygnus OB2 (cf. Green 2001) suggests that no evolved massive star has gone through the
Table 2. Summary of properties of WR 142a.

\begin{tabular}{lc}
\hline \hline$\alpha(2000)$ & $20^{\mathrm{h}} 24^{\mathrm{m}} 06.2^{\mathrm{s}}$ \\
$\delta(2000)$ & $+41^{\circ} 25^{\prime} 33^{\prime \prime}$ \\
$J$ (2MASS) & 9.22 \\
$H$ (2MASS) & 8.08 \\
$K$ (2MASS) & 7.09 \\
$J$ (June 2002) & 9.31 \\
$H$ (June 2002) & 8.15 \\
$K$ (June 2002) & 7.34 \\
spectral type & $\mathrm{WC} 8$ \\
$r(\mathrm{kpc})$ & $1.8_{-0.5}^{+0.6}$ \\
$A_{V}(\mathrm{mag})$ & $8.1 \pm 0.4$ \\
$\log \left(L / L_{\odot}\right)$ & $5.04^{1}$ \\
$M\left(M_{\odot}\right)$ & $7.9^{1}$ \\
$R\left(R_{\odot}\right)$ & $0.8^{1}$ \\
$T(\mathrm{~K})$ & $125,000^{1}$ \\
\hline
\end{tabular}

${ }^{1}$ Quantities derived from average properties of WC8 stars.

supernova phase yet. This poses some constraints on the age of Cygnus OB2. According to the evolutionary track for an initial mass of $60 M_{\odot}$ computed by Langer et al. (1994), a star enters the WC phase when its mass has decreased to $15.3 M_{\odot}$. Only when its present mass is as small as $3.92 M_{\odot}$, the star can be considered a probable candidate for a supernova explosion. This happens after $4.065 \times 10^{6}$ yrs from the mainsequence phase. Moreover, according to Langer et al. (1994), WC stars pop up around 3 million years. Hence, we may argue that Cygnus OB2 is as young as 3-4 million years, basically in agreement with Massey et al. (2001) who derived, from photometry of $\mathrm{OB}$ main-sequence stars, a mean age of $2.6 \times 10^{6} \mathrm{yrs}$.

An inspection of van der Hucht's (2001) catalogue reveals that Cygnus OB2 is the richest association in number of WC stars compared to WN stars, and the discovery of the new WC star reported here underlines this difference with respect to other associations. This could be interpreted as the result of a shallower IMF combined with the young age of the association. Indeed, an IMF slope of -0.9 has been measured for Cygnus OB2 by Massey (1998) and the cluster turn-off mass has been determined of about $120 M_{\odot}$ by Massey et al. (2001).

The discovery of a new Wolf-Rayet at a distance less than $2 \mathrm{kpc}$ from the Sun reported here dramatically illustrates the incompleteness that affects the census of even the most massive stars in our galactic neighbourhood, while stressing the need for dedicated infrared surveys to improve the sample. Such efforts will be certainly worthwhile, given the critical dependence that our understanding of the star formation history and stellar mass function in OB associations has on working on complete samples, especially when the most massive and rarest members are concerned.

Acknowledgements. We would like to thank an anonymous referee whose comments improved this manuscript. Also, it is a pleasure to thank the staff of the Calar Alto observatory, especially Mr. Santos Pedraz, for their assistance during the observing run in which the 
observations presented here were obtained. We also appreciate the help of Ms. Uta Grothkopf in making available to us the original article of Melikian and Shevchenko, and of Ms. Petia Andreeva in translating it from Russian. This paper made use of data obtained as part of the Two Micron All Sky Survey (2MASS), a joint project of the University of Massachusetts and the Infrared Processing and Analysis Center/California Institute of Technology, funded by the National Aeronautics and Space Administration and the National Science Foundation; and of the SIMBAD database, operated at CDS, Strasbourg, France.

\section{References}

Chiar, J. E., \& Tielens, A. G. G. M. 2001, ApJ, 550, 207

Cohen, M. 1995, ApJS, 100, 413

Comerón, F., Pasquali, A., Rodighiero, G., et al. 2002, A\&A, 389, 874

Comerón, F., Rieke, G. H., Claes, P., Torra, J., \& Laureijs, R. J. 1998, A\&A, 335, 522

Conti, P. S. 2000, PASP, 112, 1413

Crowther, P. A., Smith, L. J., Hillier, D. J., \& Schmutz, W. 1995a, A\&A, 293, 427

Danks, A. C., Dennefeld, M., Wamsteker, W., \& Shaver, P. A. 1983, A\&A, 118, 301

Dickel, H. R., Wendker, H., \& Bieritz, J. H. 1969, A\&A, 1, 270

Figer, D. F., McLean, I., \& Najarro, F. 1997, ApJ, 486, 420

Garmany, C. D., \& Stencel, R. E. 1992, A\&AS, 94, 211

Gervais, S., \& St-Louis, N. 1999, AJ, 118, 2394

Green, D. A. 1991, PASP, 103, 209

Green, D. A. 2001, A Catalogue of Galactic Supernova Remnants, Muller Radio Astronomy Observatory (Cambridge, UK)

Hackwell, J. A., Gehrz, R. D., \& Grasdalen, G. L. 1979, ApJ, 234, 113

van der Hucht, K. A. 2001, New Astron. Rev., 45, 135

van der Hucht, K. A., Hidayat, B., Admiranto, A. G., Supelli, K. R., \& Doom, C. 1988, A\&A, 199, 217

Knoedlseder, J. 2000, A\&A, 363, 970

Langer, N., Hamann, W.-R., Lennon, M., et al. 1994, A\&A, 290, 819

Langer, N., \& Hegel, A. 1999, in Wolf-Rayet Phenomena Stars and Starburst Galaxies, ed. K. A. van der Hucht, G. Koenigsberger, \& P. R. J. Eenens (PASP), 187
Langer, N., \& Woosley, S. E. 1996, From Stars to Galaxies: The Impact of Stellar Physics on Galaxy Evolution, ed. C. Leitherer, U. Fritze-von-Alvensleben, \& J. Huchra, ASP Conf. Ser., 98, 220

Leitherer, C. 1998, in Stellar astrophysics for the local group: VIII Canary Islands Winter School of Astrophysics, ed. A. Aparicio, A. Herrero, \& F. F. Sanchez (New York: Cambridge University Press), p. 527

Maeder, A., \& Conti, P. S. 1994, ARA\&A, 32, 227

Massey, P. 1998, in The Stellar Initial Mass Function, ed. G. Gilmore, \& D. Howell, ASP Conf. Ser., 142, 17

Massey, P., \& Conti, P. S. 1983, ApJ, 273, 576

Massey, P., DeGioia-Eastwood, K., \& Waterhouse, E. 2001, AJ, 121, 1050

Massey, P., \& Johnson, O. 1998, ApJ, 505, 793

Massey, P., Johnson, K. E., \& DeGioia-Eastwood, K. 1995, ApJ, 454, 151

Massey, P., \& Thompson, A. B. 1991, AJ, 101, 1408

Matthews, B. C., Wallace, B. J., \& Taylor, A. R. 1998, ApJ, 493, 312

Melikian, N. D., \& Shevchenko, V. S. 1990, Astrofizika, 32, 169

Meynet, G., Maeder, A., Schaller, G., Schaerer, D., \& Charbonnel, C. 1994, A\&AS, 103, 97

Miller, G. J., \& Chu, Y.-H. 1993, ApJS, 85, 137

Nugis, T., \& Lamers, H. J. G. L. M. 2000, A\&A, 360, 227

Odenwald, S. F., \& Schwartz, P. R. 1989, ApJ, 345, 470

Pineault, S., \& Terebey, S. 1997, AJ, 113, 433

Pitault, A., Epchtein, N., Gomez, A. E., \& Lortet, M. C. 1983, A\&A, 120,53

Rieke, G. H., \& Lebofsky, M. J. 1985, ApJ, 288, 618

Saken, J. M., Long, K. S., Blair, W. P., \& Winkler, P. F. 1995, ApJ, 443, 231

Schaerer, D., \& Maeder, A. 1992, A\&A, 263, 129

Schaerer, D., \& de Koter, A. 1997, A\&A, 322, 598

Shara, M. M., Moffat, A. F. J., Smith, L. F., et al. 1999, AJ, 118, 390

Smith, J. D. T., \& Houck, J. R. 2001, AJ, 121, 2115

Smith, L. F., \& Maeder, A. 1989, A\&A, 211, 71

Smith, L. F., Shara, M. M., \& Moffat, A. F. J. 1996, ApJ, 358, 229

Williams, P. M., van der Hucht, K. A., \& The, P. S. 1987, A\&A, 182, 91

Wolf, C. J. E., \& Rayet, G. 1876, Comptes Rendus, 65, 292 